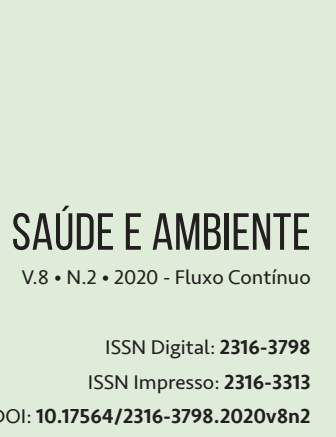

DOI: $10.17564 / 2316-3798.2020 v 8 n 2$

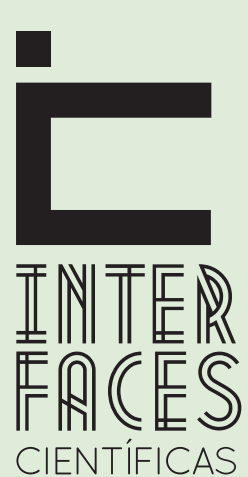

\section{PONTOS CRÍTICOS E ADEQUAÇÃO EM UNIDADE DE ALIMENTAÇÃO E NUTRIÇ̃̃O HOSPITALAR PARA SEGURANÇA DO PACIENTE}

CRITICAL POINTS AND ADEQUACY IN HOSPITAL FOOD AND NUTRITION UNIT FOR PATIENT SAFETY

PUNTOS CRIITICOS Y APTITUD EN UNA UNIDAD DE NUTRICIÓN Y ALIMENTACIÓN HOSPITALARIA PARA LA SEGURIDAD DEL PACIENTE

Maria Alice Alves Silva ${ }^{1}$ Silvia Maria Voci ${ }^{2}$ Márcia Ferreira Cândido de Souza ${ }^{3}$

Daniele Ribeiro de Souza ${ }^{4}$ Iza Maria Fraga Lobo ${ }^{5}$

\section{RESUMO}

O objetivo do estudo foi diagnosticar pontos críticos de uma Unidade de Alimentação e Nutrição Hospitalar e avaliar a taxa de adequação. Trata-se de um estudo analítico das estruturas e fluxos com diagnóstico dos pontos críticos de um serviço de nutrição e dietética de um Hospital Universitário do nordeste brasileiro, desenvolvido entre setembro de 2019 e janeiro de 2020. A etapa de avaliação (fase diagnóstica) envolveu a aplicação de um Checklist com 77 itens, baseado na RDC 275/2002 da Agência Nacional de Vigilância Sanitária (ANVISA). Os itens foram divididos em seis blocos: estruturais/organizacionais, ambiência, equipamentos/móveis, higienização, manipuladores e controles (documentações de monitoramento). Prosseguiu-se à etapa de construção do fluxograma e etapa de reavaliação e definição de pontos críticos. Os principais pontos críticos sinalizados se referem à falta de um sistema informatizado para identificação das dietas (o processo de identificação ainda é feito de forma manual) e problemas nos fluxos de entrega das refeições. A unidade de alimentação e nutrição analisada apresentou uma taxa de conformidade inicial de $68 \%$ e na reavaliação passou a ter $84 \%$ de conformidade, diferença estatisticamente significativa com valor de $\mathrm{P}>0,05$. Conclui-se que a Unidade de Alimentação e Nutrição (UAN) apresentou melhora significativa, embora ainda existam pontos críticos e falhas no processo de produção e distribuição dos alimentos que devam ser monitoradas e corrigidas para garantir a segurança alimentar do paciente.

\section{PALAVRAS-CHAVE}

Segurança Alimentar. Segurança do Paciente. Fiscalização e Controle de Instalações. 


\section{ABSTRACT}

The objective of the study was to diagnose critical points of a Hospital Food and Nutrition Unit and to evaluate the adequacy rate. This is an analytical study of the structures and flows with diagnosis of the critical points of a nutrition and dietary service at a University Hospital in northeastern Brazil, developed between September 2019 and January 2020. The evaluation stage (diagnostic phase) involved the application of a Checklist with 77 items, based on RDC 275/2002 of the National Health Surveillance Agency (ANVISA). The items were divided into six blocks: structural / organizational, ambience, equipment / furniture, hygiene, manipulators and controls (monitoring documentation). The flowchart construction stage and the reassessment and definition of critical points were continued. The main critical points signaled refer to the lack of a computerized system to identify the diets (the identification process is still done manually) and problems in the meal delivery flows. The food and nutrition unit analyzed showed an initial compliance rate of $68 \%$ and in the reevaluation it increased to $84 \%$ compliance, a statistically significant difference with a $\mathrm{P}$ value $>0.05$. It is concluded that the UAN showed significant improvement, although there are still critical points and flaws in the food production and distribution process that must be monitored and corrected to guarantee the patient's food safety.

\section{KEYWORDS}

Food safety, Patient safety, Facility Regulation and Control.

\section{RESUMEN}

El objetivo del estudio fue diagnosticar los puntos críticos de una Unidad Hospitalaria de Alimentación y Nutrición y evaluar la tasa de adecuación. Se trata de un estudio analítico de las estructuras y flujos con diagnóstico de los puntos críticos de un servicio de nutrición y dietética en un Hospital Universitario en el noreste de Brasil, desarrollado entre septiembre de 2019 y enero de 2020. La etapa de evaluación (fase de diagnóstico) involucró la aplicación de una Lista de Verificación con 77 ítems, basada en RDC 275/2002 de la Agencia Nacional de Vigilancia Sanitaria (ANVISA). Los ítems se dividieron en seis bloques: estructural/organizativo, ambiente, equipamiento/mobiliario, higiene, manipuladores y controles (documentación de seguimiento). Se continuó con la etapa de construcción del diagrama de flujo y la reevaluación y definición de puntos críticos. Los principales puntos críticos señalados se refieren a la falta de un sistema computarizado para identificar las dietas (el proceso de identificación aún se realiza de forma manual) y problemas en los flujos de entrega de comidas. La unidad de alimentación y nutrición analizada mostró una tasa de cumplimiento inicial de $68 \%$ y 
en la reevaluación aumentó a 84\% de cumplimiento, diferencia estadísticamente significativa con un valor de $\mathrm{P}>0.05$. Se concluye que la UAN mostró una mejora significativa, aunque existan puntos críticos y fallas en el proceso de producción y distribución de alimentos que deben ser monitoreados y corregidos para garantizar la seguridad alimentaria del paciente.

\section{PALABRAS CLAVE}

Seguridad alimentaria. Seguridad del paciente. Regulación y Control de Instalaciones.

\section{INTRODUCÇÃO}

Os serviços de alimentação e nutrição institucionais norteiam-se, sobretudo, para a oferta de refeições com qualidade, nutricionalmente equilibradas, seguras do ponto de vista higiênico sanitário, auxiliando na manutenção da saúde dos indivíduos (DE SETA et al., 2010). Quando os serviços de alimentação e nutrição são prestados em ambiente hospitalar, o objetivo principal torna-se a recuperação da saúde, a atenção integral ao paciente e um conjunto de cuidados. A alimentação e a nutrição em seus diferentes níveis de complexidade e de intervenção são introduzidas de acordo com as características individuais e o tipo de enfermidade (REIS et al., 2015).

Entende-se como uma alimentação de qualidade o fornecimento de alimentos íntegros, que tenham uma boa aceitação sensorial, livres de contaminantes físicos, químicos ou biológicos. Um dos fatores primordiais para a garantia da qualidade na Unidade de Alimentação e Nutrição Hospitalar (UANH) é a inocuidade do alimento em todas as etapas de produção (GERMANO, 2003). Além da oferta de alimentos seguros, a UANH também objetiva corrigir e evitar carências nutricionais que afluem o aumento das complicações e da mortalidade; identificar em tempo hábil os pacientes que requerem um maior cuidado nutricional e oferecer dietas individuais e especializadas que contribuam para amenizar a situação da doença e da internação (GARCIA, 2006).

Em 2003, o Comitê Europeu elaborou um documento contendo uma série de orientações, apelando ao envolvimento de todos os colaboradores hospitalares no sentido de se entender o trabalho do serviço de alimentação e nutrição como parte da terapêutica e da prestação de cuidados aos pacientes, referindo os cuidados a ter com a higiene e temperatura dos alimentos, fornecendo capacitações ao pessoal ligado ao serviço de alimentação, conscientizando as gestões hospitalares a incluir a segurança alimentar no âmbito mais amplo da nutrição clínica (SILVA, 2006).

Assim, na unidade hospitalar, o fornecimento de uma alimentação com qualidade juntamente com os serviços médicos e multiprofissionais, ocupam um lugar de destaque para a recuperação, manutenção e prevenção da saúde dos indivíduos internados, fazendo-se necessário estabelecer junto à cultura de segurança dos hospitais o comprometimento da gestão da saúde com a segurança alimentar do paciente a fim de melhorar a atenção à saúde (BRASIL, 2014). 
Dessa maneira o presente estudo tem objetivo de diagnosticar pontos críticos de uma unidade de alimentação e nutrição hospitalar e avaliar a sua taxa de adequação por meio da RDC 275/2002 da ANVISA. Dada a inexistência de uma resolução própria voltada para fiscalização de unidades de alimentação hospitalares, são utilizadas as RDCs voltadas para os estabelecimentos Produtores/Industrializadores de Alimentos.

\section{METODOLOGIA}

Estudo analítico das estruturas e fluxos com diagnóstico dos pontos críticos de um serviço de nutrição e dietética de um Hospital Universitário do nordeste brasileiro. Trata-se de um hospital de alta complexidade, cuja unidade de alimentação e nutrição presta serviço para 109 leitos ativos e para uma média de 150 funcionários. As refeições são distribuídas em 6 momentos: o desjejum, a colação, o almoço, o lanche da tarde, o jantar e a ceia. A seguir é descrita a composição básica do almoço e do jantar respectivamente:
ALMOÇO
I. Salada à base de vegetais crus e cozidos
II. Arroz
III. Feijão
IV. Prato principal à base de alimentos de origem animal
V. Guarnição
VI. Sobremesa (Fruta ou preparação à base de fruta)
VII. Suco de Fruta.

\section{JANTAR}
I. Salada à base de vegetais crus e cozidos
II. Arroz
III. Prato principal à base de alimentos de origem animal
IV. Guarnição
VI. Sobremesa (Fruta ou preparação a base de fruta)
VII. Suco de Fruta
OU
I. Sopa
II. Pão ou Torrada
III. Suco ou Café com Leite

Os cardápios são elaborados mensalmente e, no caso dos pacientes, são planejados de acordo com especificidades como presença de diabetes, hipertensão, problemas renais, dentre outras en- 
fermidades. A consistência das refeições também varia de líquida de prova, liquida completa, pastosa, branda e livre (Consistência padrão). A unidade também é responsável pelas dietas enterais e suplementos nutricionais.

\subsection{ETAPA DE AVALIAÇÃO - FASE DIAGNÓSTICA}

A inspeção foi realizada por meio de checklist com 77 itens divididos em seis blocos: estruturais/ organizacionais com 20 itens de avaliação, ambiência com 6 itens, equipamentos/móveis com 10 itens, higienização com 17, manipuladores com 14 e controles (documentações de monitoramento) com 10. Os itens do checklist foram retirados da Lista de Verificação das Boas Práticas de Fabricação da RDC 275/2002. Foram excluídos do checklist apenas os itens sem aplicabilidade na UANH estudada, referentes a escadas e elevadores.

No mês de dezembro de 2019, por meio de visitas diárias e observação direta, o checklist foi aplicado para avaliação e diagnóstico situacional. Utilizou-se a RDC 275/2002 da ANVISA como instrumento por ser a única resolução com lista de verificação própria para avaliação das Boas Práticas de Fabricação. Esse instrumento é validado e possui critérios que permitem a classificação das UANHS em grupos de acordo com a pontuação obtida. GRUPO I - 76 A 100\% de atendimento dos itens, GRUPO II - 51 A 75\% de atendimento dos itens, GRUPO III - 0 A 50\% de atendimento dos itens (ANVISA, 2002). 0 relatório de inconformidades observadas na etapa diagnóstica e as recomendações de adequação foi entregue à administração da unidade.

\subsection{ETAPA DE CONSTRUÇÃO DO FLUXOGRAMA E MAPEAMENTO DOS PONTOS CRÍTICOS}

Para construção do fluxograma e apontamento de etapas críticas (Aspectos higiênicos sanitários e segurança alimentar do paciente) foi realizado o mapeamento das etapas de produção e distribuição dos alimentos, bem como das dietas enterais por meio da observação direta. Os pontos críticos foram baseados no levantamento dos dados do VIGIHOSP (Software de Gestão de Riscos e Segurança do paciente e tem o objetivo de centralizar as notificações sobre incidentes ou queixas relacionadas ao paciente). Esse software é próprio do hospital e utilizou-se como filtro de busca o termo "terapia nutricional", selecionando apenas as notificações de interesse para nutrição. Também se levaram em consideração a frequência e a gravidade das notificações.

Após a etapa diagnóstica, foi produzido um relatório com todos os pontos críticos e inadequações observadas e recomendações com sugestões de cronograma, sendo o documento entregue à administração do serviço.

\subsection{ETAPA DE REAVALIAÇÃO}

Em janeiro de 2020, após o prazo de 30 dias da avaliação (etapa diagnóstica), os instrumentos de avaliação foram reaplicados e a adequação da unidade hospitalar foi reavaliada. 


\subsection{ANÁLISE DE DADOS}

As taxas de conformidade foram calculadas por meio do software OpenEpi ${ }^{\circledR}$ (versão 3.0.1), e para comparação de proporções utilizou-se o teste $\mathrm{McNemar}$ com valor de $\mathrm{p}<0,05$ para resultados significativos.

\subsection{ASPECTOS ÉTICOS}

O presente estudo é dispensado de análise por Comitê de Ética em Pesquisa com Seres Humanos e da aplicação de Termo de Consentimento Livre e esclarecido (TCLE) uma vez que não envolveu pesquisa direta com seres humanos. 0 projeto de pesquisa foi cadastrado nas bases de Ensino e Pesquisa do hospital e recebeu aprovação da chefia do setor onde foi realizada a pesquisa e do setor de Gestão da Pesquisa e Inovação Tecnológica.

\section{RESULTADOS}

O checklist aplicado continha um total de 77 itens a serem avaliados, distribuídos em seis blocos. No geral, observou-se a taxa de conformidade de $68 \%$ que classificou a UANH como GRUPO II - 51 a $75 \%$ de atendimento dos itens.

As principais não conformidades encontradas nesse primeiro momento foram referentes às estruturas e fatores organizacionais com referência ao piso, paredes, forros e portas danificadas, entulho em áreas internas e externas, ralos quebrados e utensílios em mal estado de conservação. No bloco de ambiência notou-se pouca circulação de ar na área de produção. No bloco de equipamentos e móveis foi verificado o número insuficiente de cadeiras e mesas para demanda do serviço e, por fim, no bloco de fatores de higienização pontuou-se a falta de cartazes de orientação sobre a correta higienização das mãos e o atraso na escala de higienização de equipamentos.

Após 30 dias os instrumentos foram reaplicados e a taxa de conformidade da unidade de alimentação e nutrição passou a ser 84\%, reclassificando a UANH na categoria do GRUPO I - 76 a 100\% de atendimento dos itens (Figura 1).

Figura 1 - Distribuição das taxas de conformidade da fase diagnóstica e reavaliação na Unidade de Alimentação e Nutrição Hospitalar, Sergipe, janeiro de 2020

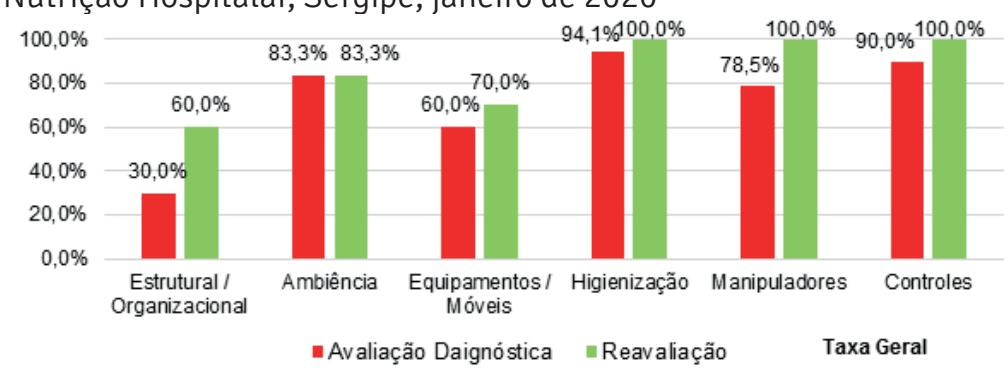

Fonte: Dados da pesquisa. 
Concomitante à aplicação do checklist foi mapeado o fluxo de preparo até a distribuição dos alimentos, sendo pontuadas seis (6) etapas críticas de controle (mais sujeitas à ocorrência de erros ou eventos relacionados à segurança alimentar do paciente).

0 ponto crítico 1 está relacionado à etapa de recebimento dos cartões que identificam as dietas. Os cartões são escritos a lápis, apagados e corrigidos muitas vezes, sinalizados por cores (Branco Dieta Branda; Azul - Dieta para hipertenso; Rosa- Dieta para diabético). Nesse processo manual pode haver erros de escrita, letras ilegíveis, confusão na interpretação da informação, erro no tipo da dieta e/ou erro de identificação do paciente.

As etapas de porcionamento e preenchimento da etiqueta da dieta, seguindo as informações dos cartões anteriores foram outras duas etapas (2 e 3 ) sinalizadas como críticas por requererem muita atenção na passagem da informação do cartão para a etiqueta, podendo ocorrer troca de identificação e dos tipos de dietas.

O ponto crítico 4 foi sinalizado na etapa de preparo e identificação da dieta do lactário. Por se tratar de uma dieta especializada e usada em pacientes mais críticos, é uma etapa que requer maior cuidado, devendo ser feita de maneira sistemática e com dupla checagem.

Por fim, as últimas etapas sinalizadas como críticas são referentes aos pontos 5 e 6 . Trata-se da chegada da alimentação na unidade clínica e administração da dieta pelo profissional adequado. Notou-se que demoras nesses processos, como por exemplo deixar a dieta muito tempo em cima da bancada da enfermaria antes de ser entregue ao paciente, tanto deixa o alimento exposto e vulnerável à contaminação como sujeito a perder suas propriedades físico-químicas (Figura 2).

Figura 2 - Fluxograma da Unidade de Alimentação e Nutrição Hospitalar (UANH): recebimento, preparo e entrega das dietas.

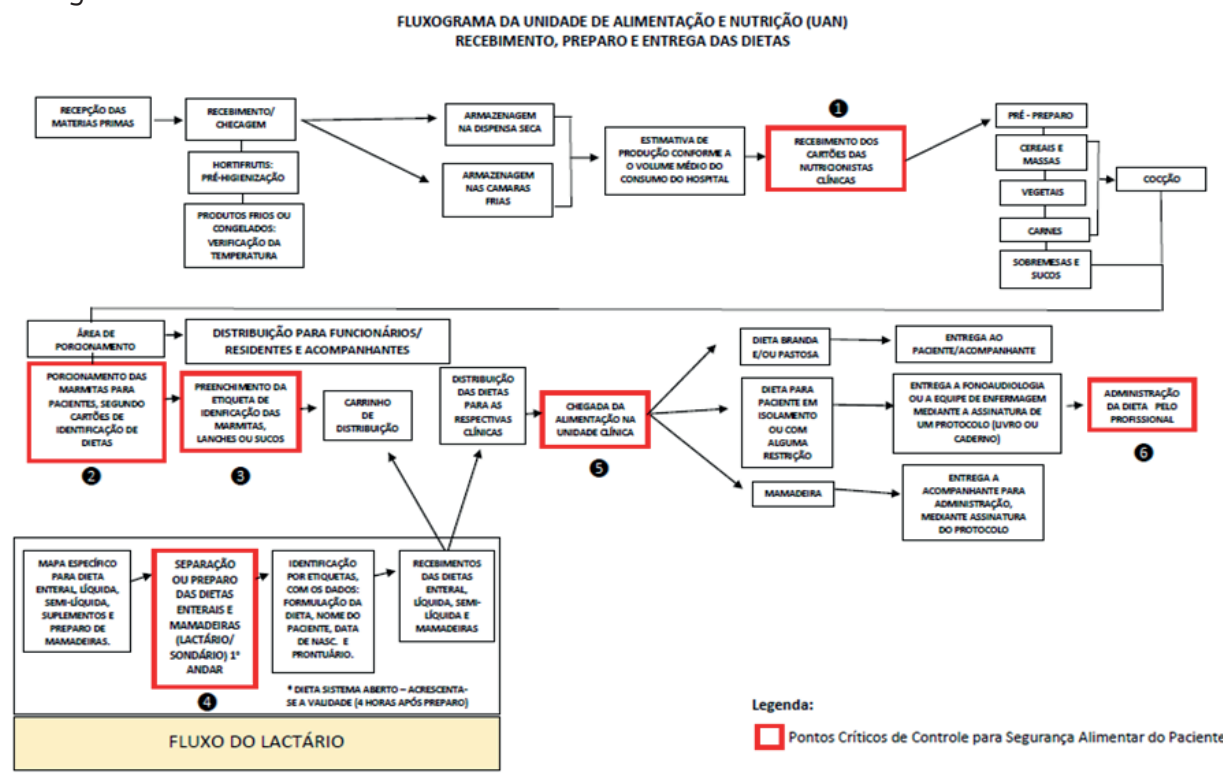

Fonte: Dados da pesquisa 


\section{DISCUSSÃO}

A UANH foi classificada na fase diagnóstica como Grupo II, cuja taxa de conformidade foi de $68 \%$ segundo o critério da RDC n 275/2002 da ANVISA, enquadrando a UANH como de média adequação. No entanto, na reavaliação passou para GRUPO I com a taxa de $84 \%$. No estudo de Vargas e colaboradores (2019), realizado em copas de distribuição de alimentos de um Serviço de Nutrição e Dietética hospitalar de Porto Alegre/Rio Grande do Sul, utilizando o mesmo instrumento de análise desta pesquisa, observou-se uma classificação de $77,7 \%$ das unidades como GRUPO I e 22,3\% como GRUPO II.

Outro resultado semelhante ao encontrado foi o realizado por Boff e Strasbourg (2018). Nele foram visitadas e inspecionadas 15 unidades de alimentação e nutrição distribuídas em grupos públicos e privados. Todas elas conseguiram atingir um nível de adequação regular, na média do GRUPO II. O percentual de adequação dos locais ficou com taxa média de 60,5\% de conformidade. Nota-se que a maioria dos estudos analisados são transversais, ao qual se aplicou o checklist, pontualmente, corroborando os dados com os da fase diagnóstica desta pesquisa, que apontam a UANH, em princípio, como média adequação.

No que diz respeito aos cuidados relacionados aos aspectos sanitários e de boas práticas, outro dado relevante são as não conformidades estruturais. Por se tratar de problemas na estrutura física torna-se difícil de ser ajustado, uma vez que demanda maior tempo, planejamento, integração multiprofissional além de um custo elevado.

Um estudo realizado em hospitais da rede pública de Brasília/DF revelou que $75 \%$ das unidades de alimentação apresentavam não conformidades nos pisos, nas paredes, nos tetos, nas portas e nas janelas (OLIVEIRA, 2008). No estudo de Fonseca e colaboradores (2010), verificou-se que vários itens referentes à edificação também estavam inadequados em relação às recomendações da RDC nº 275/2002.

Em uma UANH, deve-se fazer diariamente uma avaliação dos locais ou situações com maior probabilidade de agregar riscos para a saúde do internado e estabelecer controles para estes pontos, indicando se o alimento está dentro do esperado, ou seja, dentro da conformidade pré-planejada. Esse conjunto de medidas pode ser feito por meio da identificação dos Pontos Críticos de Controle (SOUSA, 2003).

Logo, o layout de uma UANH deve ser realizado por uma equipe multiprofissional composta por arquitetos, engenheiros, nutricionistas, administradores, dentre outros, desde a sua instalação, aquisição de equipamentos e organização até a implantação do serviço, levando em consideração a organização, os fluxos e as técnicas desenvolvidas (SILVA, 2002). Essas inadequações atrapalham os fluxos de serviços, expõem os alimentos a maiores riscos microbiológicos e predispõem o surgimento de um maior número de pontos críticos de controle.

A elaboração do fluxograma de serviço apontou nos pontos críticos de controle não só a etapa com risco de contaminação microbiológica, mas também levou em consideração as etapas mais propícias a erros, como por exemplo trocas no tipo de dieta e/ou falha na identificação correta do paciente. A qualidade do alimento é uma característica multidimensional, a qual expressa que o controle de todas as etapas do processamento de alimentos é essencial e necessita de monitoramento contínuo para assegurar a qualidade e promover a saúde ao paciente (ROCHA, 2014). 


\section{CONCLUSÃO}

Concluímos que a unidade de alimentação e nutrição analisada apresentou melhora significativa na fase de reavaliação, sendo classificada como boa adequação. O fluxograma elaborado com sinalização dos pontos críticos de controle mostrou-se importante ferramenta de controle e monitoramento para correção de possíveis falhas no processo de produção e distribuição dos alimentos e assim garantir a qualidade do alimento e fomentar a segurança da alimentação fornecida aos pacientes.

\section{REFERÊNCIAS}

ANVISA. Agência Nacional de Vigilância Sanitária. Resolução n0 275, de 21 de outubro de 2002. Disponível em: http://portal.anvisa.gov.br/documents/. Acesso em: 14 set. 2019.

BOFF, J. M.; STRASBURG, V. J. Avaliação da efetividade de boas práticas em serviços de alimentação coletiva em uma capital brasileira. Saúde, v. 44, n. 1, p. 9, 2018.

BRASIL. Ministério da Saúde. Fundação Oswaldo Cruz. Documento de referência para o programa nacional de segurança do paciente. Brasília: Ministério da Saúde. 2014.

DE SETA, M. H. et al. Cuidado nutricional em hospitais públicos de quatro estados brasileiros: contribuições da avaliação em saúde à vigilância sanitária de serviços. Ciên. Saúde Col., v. 15, p. 3413-3422, 2010.

FONSECA, M. P. et al. Avaliação das condições físico-funcionais de restaurantes comerciais para implementação das boas práticas Evaluation of physical and functional conditions of commercial restaurants to implementation of good practices. Alim. Nutr., v. 21, n. 2, p. 251-258, 2010.

GARCIA, R. W. D. A dieta hospitalar na perspectiva dos sujeitos envolvidos em sua produção e em seu planejamento. Rev. Nutr., v. 19, n. 2, p. 129-144, 2006.

GERMANO, M. I. S. Treinamento de manipuladores de alimentos: fator de segurança alimentar e promoção da saúde. In: GERMANO, M. I. S. Treinamento de manipuladores de alimentos: fator de segurança alimentar e promoçäo da saúde. São Paulo: Editora Varela. 2003.

OLIVEIRA, M. N. Avaliação das condições higiênico-sanitárias das cozinhas de creches públicas e filantrópicas. Ciên. Saúde Col., v. 13, p. 1051-1060, 2008. 
REIS, H. F. et al. Avaliação das condições higiênico-sanitárias de uma unidade de alimentação e nutrição hospitalar de Montes Claros, MG. Unimontes Cient., v. 17, n. 2, p. 68-81, 2015.

ROCHA, A. A. Avaliação da estrutura e dos processos hospitalares para melhoria da qualidade na assistência. 2014. 123 f. Dissertação (Mestrado Profissional) - Universidade Federal dos Vales do Jequitinhonha e Mucuri, Diamantina, 2014.

SILVA, E. A. J. Manual de controle higiênico-sanitário em alimentos. São Paulo: Editora Varela, 2002.

SILVA, N. M. Diagnóstico situacional das ferramentas de segurança na produção de alimentos das unidades de alimentação e nutrição dos Hospitais de Brasília-DF. 2006. 107 f. Dissertação (Mestrado) - Universidade de Brasília, Brasília, 2006.

SOUSA, C. L.; CAMPOS, G. D. Condições higiênico-sanitárias de uma dieta hospitalar. Rev. Nutr., v. 16, n. 1, p. 127-134, 2003.

VARGAS, F. S. et al. Avaliação da adequação às boas práticas em serviço de nutrição de um hospital universitário de Porto Alegre/RS. Disciplinarum Sci. Série: Ciên. Saúde, v. 19, n. 3, p. 549-557, 2018. 
1 Residência Multiprofissional em Epidemiologia Hospitalar, Hospital Universitário de Sergipe, Universidade Federal de Sergipe - UFS; Nutricionista.

E-mail: alicealvesnutri@hotmail.com

2 Doutora em Nutrição em Saúde Pública. Professora do Departamento de Nutrição, Centro de Ciências Biológicas e da Saúde, Universidade Federal de Sergipe - UFS; Nutricionista. E-mail: smvoci2@gmail.com

3 Nutricionista; Docente da Unidade de Alimentação e Nutrição, Hospital Universitário de Sergipe e do Programa de Mestrado Profissional em Gestão e Inovação Tecnológica em Saúde da Universidade Federal de Sergipe - UFS. E-mail: nutrimarciacandido@gmail.com

4 Residência Multiprofissional em Epidemiologia Hospitalar, Hospital Universitário de Sergipe, Universidade Federal de Sergipe - UFS; Enfermeira, Secretaria de Saúde do Estado da Bahia. E-mail: daniiers@hotmail.com

5 Doutorado em Medicina e Saúde, Gerência de Risco, Hospital Universitário, Universidade Federal de Sergipe UFS; Médica. E-mail: izalobo@globo.com

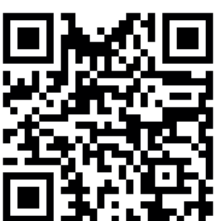

A autenticidade desse artigo pode ser conferida no site https://periodicos. set.edu.br

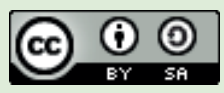

Este artigo é licenciado na modalidade acesso aberto sob a Atribuição-Compartilha Igual CC BY-SA

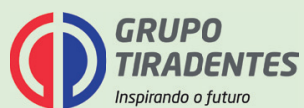

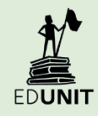

